\title{
Drug-induced hemolytic crisis during ibrutinib plus venetoclax therapy for the treatment of Mantle-cell lym- phoma: A rare hematologic adverse reaction
}

\author{
Xin Wen ${ }^{\mathrm{a}}$, Yingzhi He ${ }^{\mathrm{b}}$, Shan Wang ${ }^{\mathrm{c}}$, Liang Wang ${ }^{\mathrm{d}, *}$ \\ ${ }^{a}$ Department of Pharmacy, ZhuJiang Hospital of Southern Medical University, Guangzhou, China. \\ ${ }^{b}$ Department of Hematology, ZhuJiang Hospital of Southern Medical University, Guangzhou, China. \\ ${ }^{c}$ Department of Pharmacy, Winthrop-University Hospital, Mineola, NY, USA. \\ ${ }^{d}$ Department of Hematology, Beijing Tongren Hospital, Capital Medical University, Beijing, China.
}

\begin{abstract}
With the increasing development of novel targeted agents, several subtypes of lymphoma are entering the chemotherapy-free era. The combination of Bruton's tyrosine kinase inhibitor ibrutinib and the BCL-2 inhibitor venetoclax has been proven to be highly effective in the treatment of relapsed/refractory mantle cell lymphoma (MCL). The most common adverse effects of this combined therapy are gastrointestinal, such as diarrhea, nausea or vomiting, and gastroesophageal reflux. However, hemolysis is a rare event. In this paper, we report a case of a 50-year-old MCL patient who experienced a hemolytic crisis during treatment with ibrutinib and venetoclax. The case demonstrates a rare but potentially lethal adverse event and emphasizes the need for suspicion of such an adverse reaction during this combination therapy.
\end{abstract}

Keywords: Mantle cell lymphoma, hemolytic crisis, ibrutinib, venetoclax

\section{Introduction}

Mantle cell lymphoma (MCL) is an uncommon subtype of non-Hodgkin lymphoma (NHL). Though it comprises only $6 \%$ of NHL, MCL is a clinical challenge according to its aggressive clinical behavior and poor outcomes [1]. Ibrutinib is a first-in-class covalent and irreversible Bruton's tyrosine kinase (BTK) inhibitor that has demonstrated activity in several B-cell malignancies [2]. Venetoclax is a highly selective inhibitor of BCL-2, an antiapoptotic protein that has elevated expression in many hematologic malignancies, including MCL $[3,4]$. The targeting of BTK and BCL-2 with ibrutinib and venetoclax is the most effective drug approach in the treatment of chronic lymphocytic leukemia (CLL) and relapsed/refractory MCL [5, $6]$. The combined therapy of ibrutinib and venetoclax is an

\footnotetext{
* Corresponding author: Liang Wang

Mailing address: Department of Hematology, Beijing Tongren Hospital, Capital Medical University, Beijing, China.

Email: wangliangtrhos@126.com

Received: 06 December 2019 / Accepted: 07 December 2019
}

innovative strategy and is consistent with favorable therapeutic effectiveness in relapsed/refractory MCL patients who have a poor prognosis with current treatment. The most common adverse events of this combined therapy are gastrointestinal, such as diarrhea, nausea or vomiting, and gastroesophageal reflux. Tumor lysis syndrome is a common serious adverse event [5].

\section{Case report}

A 50-year-old male patient was diagnosed with MCL (stage IV, simplified MIPI score=3) in 2011, and the firstline therapy was six cycles of R-hyperCVAD/R-MA; the complete response (CR) duration lasted for 24 months. In 2013, his disease relapsed and was treated with rituximab+bortezomib+high-dose cytarabine for three cycles as the second-line therapy. Though CR was attained, the collection of autologous stem cells failed, and thalidomide maintenance was initiated until the disease relapsed later in 2015. The second progression-free survival (PFS) period was 32 months. Then, two cycles of R-CHOP/RDHAP were given followed by lenalidomide maintenance, and the disease relapsed for the third time in 2017 (PFS $=26$ months). The patient developed pancytopenia and a 
Table 1. Naranjo scale for the likelihood of ibrutinib plus venetoclax-induced AIHA.

\begin{tabular}{|c|c|c|c|c|}
\hline Question & Yes & No & Don't know & Score \\
\hline 1. Are there previous conclusive reports on this reaction? & +1 & 0 & 0 & 0 \\
\hline 2. Did the adverse event appear after the suspected drug was administered? & +2 & -1 & 0 & +2 \\
\hline $\begin{array}{l}\text { 3. Did the adverse reaction improve when the drug was discontinued or a specific antagonist } \\
\text { was administered? }\end{array}$ & +1 & 0 & 0 & +1 \\
\hline 4. Did the adverse reaction reappear when the drug was re-administered? & +2 & -1 & 0 & 0 \\
\hline $\begin{array}{l}\text { 5. Are there possible alternative causes that could have caused the reaction? Are there alternate } \\
\text { causes (other than the drug) that could have solely caused the reaction? }\end{array}$ & -1 & +2 & 0 & +2 \\
\hline 6. Did the reaction reappear when a placebo was given? & -1 & +1 & 0 & 0 \\
\hline 7. Was the drug detected in the blood (or other fluids) in concentrations known to be toxic? & +1 & 0 & 0 & 0 \\
\hline $\begin{array}{l}\text { 8. Was the reaction more severe when the dose was increased or less severe when the dose } \\
\text { was decreased? }\end{array}$ & +1 & 0 & 0 & +1 \\
\hline 9. Did the patient have a similar reaction to the same or similar drugs in any previous exposure? & +1 & 0 & 0 & 0 \\
\hline 10. Was the adverse event confirmed by any objective evidence? & +1 & 0 & 0 & 0 \\
\hline Total score & & & & 6 \\
\hline
\end{tabular}

high tumor burden at this time, and ibrutinib monotherapy was administrated at a dose of $560 \mathrm{mg}$ per day. Two months later, CR was confirmed by both positron emission tomography-computed tomography (PET-CT) scan and bone marrow biopsy, but the disease relapsed seven months later in 2018 with significant bone marrow infiltration. Next generation sequencing using a bone marrow specimen did not reveal any mutations involving BTK, CARD11, CD79b, MYD88, or TP53. Venetoclax was given according to a dosing schedule that started at $100 \mathrm{mg}$ per day and increased by $100 \mathrm{mg}$ daily to reach $600 \mathrm{mg}$ per day. Partial remission (PR) was attained two months later; however, venetoclax was discontinued due to the patient's economic status, and the disease progressed five months after the PR (PFS $=7$ months). After treatment with ibrutinib or venetoclax, we monitored the patient using a Coombs' test periodically, and the results of a direct antiglobulin test showed negative anti-immunoglobulin $\mathrm{G}$ (IgG) and anti-complement C3 (anti-C3).

In May 2019, the patient suffered from aggressive progression of MCL, and a PET-CT scan revealed multi-lymphoadenopthy and splenomegaly. As shown in Figure 1, the patient began with ibrutinib at $560 \mathrm{mg}$ per day orally, and venetoclax was introduced the next day according to a dosage schedule that started at $100 \mathrm{mg}$ per day and gradually increased by $100 \mathrm{mg}$ daily to reach $600 \mathrm{mg}$ per day. After two days of this full-dose combination of ibrutinib and venetoclax, the patient presented to our clinic with dizziness, severe fatigue, pallor, and soy-sauce colored urine. Laboratory test results showed an extremely low level of hemoglobin $(28.0 \mathrm{~g} / \mathrm{L})$ and a significant increase in the reticulocyte count $(19.5 \%)$, total bilirubin (TBIL $3.567 \mathrm{mg} / \mathrm{dL})$, and indirect bilirubin $(3.029 \mathrm{mg} / \mathrm{dL})$. A Coombs' test was positive for both anti-IgG and anti-C3, indicating the diagnosis of severe autoimmune hemolytic anemia (AIHA). The hemolytic crisis was attributed to the high-dose combination of ibrutinib plus venetoclax and most likely the rapid titration of venetoclax. The probability of an adverse drug reaction (ADR) was assessed using the Naranjo algorithm (Table 1) [7]. It revealed a probable ADR score of +6 .

Therefore, this combined therapy was immediately discontinued, and an intravenous infusion of methylprednisolone
(1000 mg daily for three days), immunoglobulin (IVIg) at $25 \mathrm{~g}$ for five days, and washed red blood cells were given. Afterward, the dose of methylprednisolone was halved and maintained for three days, and then $250 \mathrm{mg}$ was given for four days. Thereafter, the methylprednisolone was reduced to $80 \mathrm{mg}$ for four days followed by $40 \mathrm{mg}$ for three days. Oral administration of $32 \mathrm{mg}$ once daily for three days was then administered and stepped down to 8 mg weekly until a maintained dose of $8 \mathrm{mg}$ per day was reached. The adverse AIHA event was alleviated according to all parameters; hemoglobin, reticulocyte count, and bilirubin returned to normal. One month after the onset of the hemolytic crisis, anti-MCL treatment was reinitiated while the patient was still taking methylprednisolone $8 \mathrm{mg}$ orally daily. At that time, ibrutinib monotherapy at a dose of $280 \mathrm{mg}$ per day was given for the first week, then venetoclax was administrated $100 \mathrm{mg}$ per day in the second week and increased weekly by $100 \mathrm{mg}$ per day. At the time of this writing, the patient is receiving $280 \mathrm{mg} / \mathrm{d}$ ibrutinib and $300 \mathrm{mg} / \mathrm{d}$ venetoclax daily and is not experiencing any adverse reactions like AIHA ( Figure 1).

\section{Discussion}

This is the first report of a severe AIHA secondary to the combination therapy of ibrutinib and venetoclax in MCL. AIHA is known to complicate CLL and occurs in up to $30 \%$ of patients but is uncommon in aggressive NHL, with an estimated incidence of 1\% [8]. Drug-induced immune hemolytic anemia can be classified into an autoimmune type, a drug adsorption type, and a neoantigen type [9]. Depending on the number of reports in the literature, the drugs that are most associated with AIHA are the new generation of cephalosporins, diclofenac, oxaliplatin, and fludarabin [10].

High-dose glucocorticoids are considered the first-line treatment for AIHA. If AIHA is a potential complication of MCL, patients should be more successfully treated by focusing on the underlying lymphoma instead of relying on steroids [11]. In this case, both ibrutinib and venetoclax were discontinued immediately at the onset of AIHA, while a high dose of glucocorticoid was initiated 


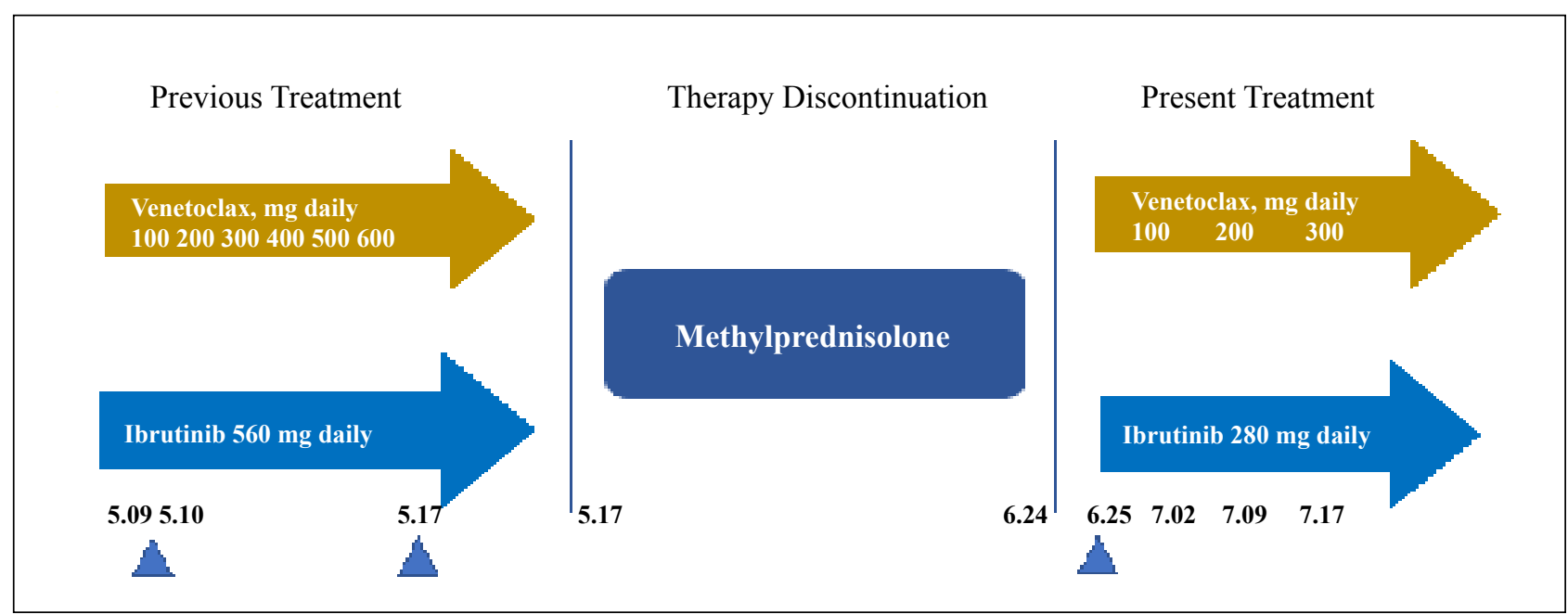

Figure 1. The treatment process of MCL and the hemolytic crisis.

to reverse the AIHA. Thus, it can be inferred that AIHA was induced by the targeted therapy of ibrutinib and venetoclax rather than being a complication of the underlying MCL.

Molica and Polliack [12] believed that ibrutinib could be effective in a variety of immune disorders and also suggested that the acute AIHA that appeared in the CLL treatment with ibrutinib was caused by CLL activity rather than an ibrutinib-induced reaction. Although a preclinical study indicated that ibrutinib may inhibit the production of autoantibodies [13], the detailed mechanism of action of ibrutinib in AIHA needs to be further investigated. AIHA has been reported as one of the adverse events in relapsed/ refractory CLL patients treated with venetoclax [14], but it is rarely reported in patients with MCL.

In this case, we consider that the high-dose combination therapy and rapid titration process of venetoclax may have contributed to the development of severe AIHA. In the study conducted by Tam C.S et al. [5], to reduce the risk of tumor lysis syndrome, MCL patients took $560 \mathrm{mg}$ ibrutinib daily first. After four weeks, venetoclax was added in a step-wise fashion, increasing each week from $100 \mathrm{mg}$ per day up to the maximum dose of $400 \mathrm{mg}$ daily. AIHA was not reported in that study.

\section{Conclusion}

Since ibrutinib and venetoclax are prescribed with increasing frequency for various types of hematologic malignancies, physicians should be aware of the rare but serious complication of AIHA.

\section{Declarations}

Ethical approval: This article does not contain any studies with human participants performed by any of the authors.
Conflict of Interest: Liang Wang is a member of the Editorial Board of Aging Pathobiology and Therapeutics. All authors declare no conflict of interest and were not involved in the journal's review or desicions related to this manuscript.

Funding: This study was funded by the National Natural Science Foundation of China (81400159, 81873450), and Pearl River Nova Program of Guangzhou (201710010161).

Informed consent: Informed consent was obtained from all individual participants included in the study.

\section{References}

1. Cheah CY, Seymour JF, Wang ML. Mantle Cell Lymphoma. Journal of Clinical Oncology, 2016,34(11):1256-69.

2. Wang $M$ L, Lee $H$, Chuang $H$, et al. Ibrutinib in combination with rituximab in relapsed or refractory mantle cell lymphoma: a single-centre, open-label, phase 2 trial. The Lancet Oncology, 2016, 17(1): 48-56.

3. Waldron M, Winter A, Hill B T. Pharmacokinetic and pharmacodynamic considerations in the treatment of chronic lymphocytic leukemia: ibrutinib, idelalisib, and venetoclax[J]. Clinical pharmacokinetics, 2017, 56(11): 1255-1266.

4. Lampson B L, Davids M S. A new triple threat to CLL. Blood, The Journal of the American Society of Hematology, 2018, 132(15): 1547-1548.

5. Tam C S, Anderson M A, Pott C, et al. Ibrutinib plus venetoclax for the treatment of mantle-cell lymphoma. New England Journal of Medicine, 2018, 378(13): 1211-1223.

6. Jain N, Keating M, Thompson P, et al. Ibrutinib and venetoclax for first-line treatment of CLL. New England Journal of Medicine, 2019, 380(22): 2095-2103.

7. Naranjo C A, Busto U, Sellers E M, et al. A method for estimating the probability of adverse drug reactions. Clinical Pharmacology \& Therapeutics, 1981, 30(2): 239-245.

8. Doni E, Carli G, Di Rocco A, et al. Autoimmune haemolytic 
anaemia in mantle cell lymphoma: an insidious complication associated with leukemic disease. Hematological oncology, 2017, 35(1): 135-137.

9. Gehrs B C, Friedberg R C. Autoimmune hemolytic anemia. American journal of hematology, 2002, 69(4): 258271.

10. Salama A. Drug-induced immune hemolytic anemia. Expert opinion on drug safety, 2009, 8(1): 73-79.

11. Eve H E, Rule S A J. Autoimmune haemolytic anaemia associated with mantle cell lymphoma. International journal of hematology, 2010, 91(2): 322-325.
12. Molica S, Polliack A. Autoimmune hemolytic anemia (AIHA) associated with chronic lymphocytic leukemia in the current era of targeted therapy. Leukemia research, 2016, 50: 31-36.

13. Beckering G, Zilker S J, Haarer D. Spectral measurements of the emission from highly scattering gain media. Optics letters, 1997, 22(18): 1427-1429.

14. Davids M S, Hallek M, Wierda W, et al. Comprehensive safety analysis of venetoclax monotherapy for patients with relapsed/refractory chronic lymphocytic leukemia. Clinical Cancer Research, 2018, 24(18): 4371-4379.

Cite this article as: Wen X, He Y, Wang S, et al. Drug-Induced Hemolytic Crisis During Ibrutinib plus Venetoclax for the Treatment of Mantle-Cell Lymphoma: A Rare Hematologic Adverse Reaction [J]. Aging Pathobiology and Therapeutics, 2019, 1(1): 25-28. 